\title{
Estimates of Approximation Error by Legendre Wavelet
}

\author{
Xiaoyang Zheng, Zhengyuan Wei \\ College of Mathematics and Statistics, Chongqing University of Technology, Chongqing, China \\ Email: zhengxiaoyang@cqut.edu.cn
}

Received 10 March 2016; accepted 25 April 2016; published 28 April 2016

Copyright (C) 2016 by authors and Scientific Research Publishing Inc.

This work is licensed under the Creative Commons Attribution International License (CC BY).

http://creativecommons.org/licenses/by/4.0/

(c) () Open Access

\begin{abstract}
This paper first introduces Legendre wavelet bases and derives their rich properties. Then these properties are applied to estimation of approximation error upper bounded in spaces $C^{\alpha}([0,1])$ and $C^{N+\alpha}([0,1])$ by norms $\|\cdot\|_{2}$ and $\|\cdot\|_{1}$, respectively. These estimate results are valuable to solve integral-differential equations by Legendre wavelet method.
\end{abstract}

Keywords

Legendre Wavelet, Estimate, Exponential $\alpha$-Hölder Continuity

\section{Introduction}

In recent years, an application of Legendre wavelet to solve integral-differential equations and partial differential equations is deeply considered [1]-[9]. Generally, representations of function and operator by Legendre wavelet are exact up to arbitrary but finite precision, then the approximation error should be estimated. Although estimating the approximation error is a tough technique, if the wavelet satisfies certain conditions [5]-[11], then the upper bounded of the wavelet transform coefficients can be estimated. In this article, we use the rich properties of Legendre wavelet bases such as compactly supported, polynomials, orthogonality to estimate the approximation error upper bounded.

In this paper, Section 2 introduces Legendre wavelet bases and its properties. Section 3 estimates the approximation error upper bounded by norms \|\|$_{2}$ and $\|\cdot\|_{1}$ for spaces $C^{\alpha}([0,1])$ and $C^{N+\alpha}([0,1])$, respectively. This paper ends with brief conclusion.

\section{Legendre Wavelet and Its Properties}

In this section, we first briefly introduce Legendre wavelet bases and our notations. Secondly, the rich properties 
and some important results of Legendre wavelet that will be used later are elaborated.

\subsection{Legendre Wavelet}

For level of decomposition $n=0,1, \cdots$ and translation $l=0,1,2, \cdots, 2^{n}-1$, we define subinterval $I_{n l}=\left[2^{-n} l, 2^{-n}(l+1)\right)$. For $p=1,2, \cdots$, define $V_{p, n}$ as a subspace of piecewise polynomial functions satisfying

$$
V_{p, n}=\left\{f:\left.f\right|_{I_{n l}} \text { is a polynomial of degree strictly less than } p \text { and } f \text { vanishes elsewhere }\right\} \text {. }
$$

We now start to review Legendre polynomials and Legendre wavelet bases [1]. Let $L_{k}(x)$ denote Legendre polynomial of degree $k$, which is defined as follows:

$$
\begin{aligned}
& L_{1}(x)=x, L_{1}(x)=x, \\
& L_{k+2}(x)=\frac{2 k+3}{k+2} x L_{k+1}(x)-\frac{k+1}{k+2} L_{k}(x) .
\end{aligned}
$$

Then, at the level of resolution $n=0$, let $\phi_{k}(x)$ denote Legendre wavelet bases defined as

$$
\phi_{k}(x)= \begin{cases}\sqrt{2 k+1} L_{k}(2 x-1), & x \in[0,1], \\ 0, & x \notin[0,1] .\end{cases}
$$

The whole set $\left\{\phi_{k}\right\}_{k=0}^{p-1}$ forms an orthonormal basis for $V_{p, 0}$. Generally, the subspace $V_{p, n}$ is spanned by $2^{n} p$ functions which are obtained from $\phi_{0}, \cdots, \phi_{k-1}$ by dilation and translation, i.e.,

$$
V_{p, n}:=V_{p, n l}=\operatorname{span}\left\{\phi_{k, n l}(x)=2^{n / 2} \phi_{k}\left(2^{n} x-l\right), 0 \leq k \leq p-1,0 \leq l \leq 2^{n}-1\right\}
$$

which forms an orthonormal basis for $L_{2}([0,1])$ and

$$
V_{p, 0} \subset V_{p, 1} \subset \cdots \subset V_{p, n} \subset \cdots .
$$

Now, let $p=3, n=1$, then obtain six Legendre wavelet base functions which are given by

$$
\begin{aligned}
& \phi_{0,10}(x)=\sqrt{2} \text {, } \\
& \phi_{1,10}(x)=\sqrt{6}(4 x-1) \\
& \phi_{2,10}(x)=\sqrt{10}\left[\frac{3}{2}(4 x-1)^{2}-\frac{1}{2}\right], \quad 0 \leq x<\frac{1}{2} \\
& \phi_{0,11}(x)=\sqrt{2}, \quad \phi_{1,11}(x)=\sqrt{6}(4 x-3) \\
& \phi_{2,11}(x)=\sqrt{10}\left[\frac{3}{2}(4 x-3)^{2}-\frac{1}{2}\right], \quad \frac{1}{2} \leq x<1
\end{aligned}
$$

and Figure 1 illustrates these base function as

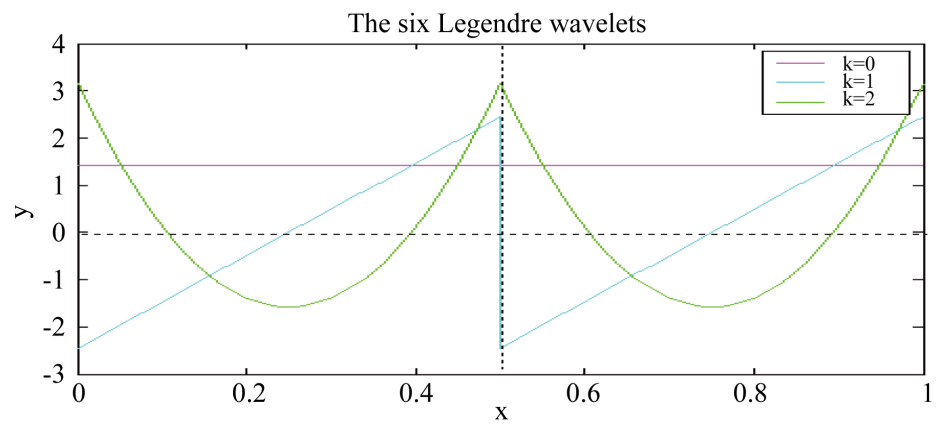

Figure 1. The six Legendre wavelet bases with $k=0,1,2 ; n=1$. 


\subsection{Some Properties of Legendre Wavelet}

It is clear that Legendre wavelet bases are compactly supported, polynomial, bounded and orthogonal on each subinterval $I_{n l}$. These properties are very useful to estimate the approximation error upper bounded.

Lemma 1. Legendre wavelet bases satisfy the results

$$
\phi_{k, n l}\left(l / 2^{n}\right)=(-1)^{k} \sqrt{4 k+2}, \quad \phi_{k, n l}\left((l+1) / 2^{n}\right)=\sqrt{4 k+2} .
$$

Lemma 2. For any $x \in[0,1)$, Legendre wavelet $\phi_{k, n l}(x)$ are bounded by the form

$$
\left|\phi_{k, n l}(x)\right| \leq \sqrt{4 k+2}
$$

where $k$ is the order of Legendre wavelet.

Proof. According to the definition of Legendre wavelet bases, Legendre wavelet defined on subinterval $I_{n l}$ are obtained through Legendre polynomials by dilation. With the result of Legendre polynomials $\left|L_{k}(x)\right| \leq 1$, the bound of Legendre wavelet is easily proved.

Lemma 3. A relation of between Legendre wavelet and their derivative on each subinterval $I_{n l}$ is derived as

$$
\sqrt{2 k+3} \phi_{k+1, n l}(x)=\frac{1}{\sqrt{2 k+5}} \phi_{k+2, n l}^{\prime}(x)-\frac{1}{\sqrt{2 k+1}} \phi_{k, n l}^{\prime}(x) .
$$

Proof. Using the result of between Legendre polynomials and their derivative, i.e.,

$$
(2 k+3) L_{k+1}(x)=L_{k+2}^{\prime}(x)-L_{k}^{\prime}(x),
$$

we can obtain the above result.

Using this result, we can obtain

$$
\begin{aligned}
\int_{l / 2^{n}}^{(l+1) / 2^{n}} \phi_{k+1, n l}(x) \mathrm{d} x= & \frac{1}{\sqrt{(2 k+3)(2 k+5)}} \int_{l / 2^{n} / 2^{n}}^{(1+1) / \phi_{k+2, n l}^{\prime}}(x) \mathrm{d} x \\
& -\frac{1}{\sqrt{(2 k+3)(2 k+1)}} \int_{l / 2^{n}}^{(l+1) / 2^{n}} \phi_{k, n l}^{\prime}(x) \mathrm{d} x \\
= & \frac{1}{\sqrt{(2 k+3)(2 k+5)}}\left[\sqrt{4 k+10}-(-1)^{k} \sqrt{4 k+10}\right] \\
& -\frac{1}{\sqrt{(2 k+3)(2 k+1)}}\left[\sqrt{4 k+2}-(-1)^{k} \sqrt{4 k+2}\right] \\
= & 0 .
\end{aligned}
$$

However, when $k=0$, the integration is calculated as

$$
\int_{l / 2^{n}}^{(l+1) / 2^{n}} \phi_{0, n l}(x) \mathrm{d} x=\sqrt{2} / 2^{n} .
$$

Now, the orthogonal property of Legendre wavelet bases is given by

Lemma 4. Legendre wavelet bases defined on the interval $[0,1)$ are orthogonal.

Proof. According to the compactly supported of Legendre wavelet bases, we know that any two such base functions $\phi_{k, n l}(x)$ and $\phi_{k^{\prime}, n l^{\prime}}(x)$ with the same scale index $n$ and different $k, k^{\prime}, l, l^{\prime}$ are orthogonal. If any two bases functions are only different in $k, k^{\prime}$, then for any $x \in I_{n m}$, we have

$$
\begin{aligned}
& \int_{l / 2^{n}}^{(l+1) / 2^{n}} \phi_{k, n l}(x) \phi_{k^{\prime}, n l}(x) \mathrm{d} x \\
& =\int_{l / 2^{n}}^{(l+1) / 2^{n}}(2 k+1)^{1 / 2} 2^{n / 2} L_{k}\left(2^{n+1} x-2 l-1\right)\left(2 k^{\prime}+1\right)^{1 / 2} 2^{n / 2} L_{k^{\prime}}\left(2^{n+1} x-2 l-1\right) \mathrm{d} x \\
& =(2 k+1)^{1 / 2}\left(2 k^{\prime}+1\right)^{1 / 2} / 2 \int_{-1}^{1} L_{k}(v) L_{k^{\prime}}(v) \mathrm{d} v=0
\end{aligned}
$$

which completes the proof.

Thus, any function $f(x)$ belonging to $L_{2}([0,1])$ can be expanded as 


$$
f(x)=\lim _{n \rightarrow \infty} \sum_{k=0}^{\infty} \sum_{l=0}^{\infty} s_{k, n l} \phi_{k, n l}(x),
$$

where $s_{k, n l}=\left(f(x), \phi_{k, n l}(x)\right)$ is Legendre wavelet coefficients and (...) denotes inner product. Accordingly, norm equality is given by

$$
\|f\|_{2}=\lim _{n \rightarrow \infty} \sum_{k=0}^{\infty} \sum_{l=0}^{\infty}\left(s_{k, n l}\right)^{2} .
$$

If approximation of the function is analyzed in the space $V_{p n}$, then the approximation formula is described by

$$
f(x) \approx \sum_{k=0}^{p-1} \sum_{l=0}^{2^{n}-1} s_{k, n l} \phi_{k, n l}(x)=S^{\mathrm{T}} \Phi(x),
$$

where $S$ and $\Phi(x)$ are $2^{n} p \times 1$ matrices and defined as, respectively

$$
\begin{gathered}
S=\left[s_{0, n 0}, s_{1, n 0}, \cdots, s_{p-1, n 0}, s_{0, n 1}, s_{1, n 1}, \cdots, s_{p-1, n 1}, \cdots, s_{0, n\left(2^{n}-1\right)}, s_{1, n\left(2^{n}-1\right)}, \cdots, s_{p-1, n\left(2^{n}-1\right)}\right]^{\mathrm{T}}, \\
\Phi(x)=\left[\phi_{0, n 0}, \phi_{1, n 0}, \cdots, \phi_{p-1, n 0}, \phi_{0, n 1}, \phi_{1, n 1}, \cdots, \phi_{p-1, n 1}, \cdots, \phi_{0, n\left(2^{n}-1\right)}, \phi_{1, n\left(2^{n}-1\right)}, \cdots, \phi_{p-1, n\left(2^{n}-1\right)}\right]^{\mathrm{T}}
\end{gathered}
$$

which makes the function approximated by arbitrary precision, when numerical computation is adopted by Legendre wavelet method.

\section{Upper Bounded Estimates of Approximation Error by Legendre Wavelet}

In this section, the preliminaries of the function spaces with respect to exponential $\alpha$-Hölder continuity and $C^{N+\alpha}([0,1])$ are first introduced, respectively. Secondly, the upper bounded estimates of approximation error in the spaces by Legendre wavelet bases are derived by norms $\|\cdot\|_{2}$ and $\|\cdot\|_{1}$, respectively.

\subsection{Exponential $\alpha$-Hölder Continuity and $C^{N+\alpha}([0,1])$}

The preliminaries of exponential $\alpha$-Hölder continuity $C^{\alpha}([0,1])$ and $C^{N+\alpha}([0,1])$ spaces are defined by

Definition 1. Exponential $\alpha$-Hölder continuity for any $\alpha(0<\alpha \leq 1)$ denotes the function $f$ satisfying

$$
|f(x)-f(y)| \leq A|x-y|^{\alpha}, \forall x, y \in R
$$

for some positive constant $A$.

Definition 2. $C^{N+\alpha}(R)$ space denotes that all the functions $f$ which are bounded and continuously differentiable up to $N$-order for any $\alpha(0<\alpha \leq 1)$, i.e.,

$$
C^{N+\alpha}(R)=\left\{f \mid \sup _{\substack{x, y \in R \\ x \neq y}} \frac{\left|f^{\left(k_{0}\right)}(x)-f^{\left(k_{0}\right)}(y)\right|}{|x-y|^{\alpha}}<\infty\right\}
$$

where $k_{0}=0,1, \cdots, N$.

\subsection{Approximation Error Estimate by Norm \|\|$_{2}$}

The upper bounded of Legendre wavelet transform coefficients is estimated as:

Theorem 1. Let $f \in C^{\alpha}([0,1])$, then the upper bounded estimate of Legendre wavelet transform coefficients satisfies

$$
\left|s_{k, n l}\right| \leq T_{k f l} 2^{-v}, v=\min \{(n+1) \alpha, n\}
$$

where $T_{k f l}$ is a constant with respect to $k$, $f$ and $l$.

Proof. Taking advantage of the results of (6) and (7), we have 


$$
\begin{aligned}
\left|s_{k, n l}\right| & =2^{n / 2} \sqrt{2 k+1}\left|\int_{l / 2^{2^{n}}}^{(l+1) / 2^{n}}\left[f(x)-f\left((2 l+1) / 2^{n+1}\right)+f\left((2 l+1) / 2^{n+1}\right)\right] L_{k}\left(2^{n+1} x-2 l-1\right) \mathrm{d} x\right| \\
& \leq 2^{n / 2} \sqrt{2 k+1}\left[\int_{l / 2^{n}}^{(l+1) / 2^{n}}\left|f(x)-f\left((2 l+1) / 2^{n+1}\right)\right| \mathrm{d} x+\left|\int_{l / 2^{n}}^{(l+1) / 2^{n}} f\left((2 l+1) / 2^{n+1}\right) L_{k}\left(2^{n+1} x-2 l-1\right) \mathrm{d} x\right|\right] \\
& \leq 2^{n / 2} \sqrt{2 k+1}\left[\int_{l / 2^{n}}^{(l+1) / 2^{n}}\left|x-(2 l+1) / 2^{n+1}\right|^{\alpha} d x+\left|f\left((2 l+1) / 2^{n+1}\right)\right|\left|\int_{l / 2^{n}}^{(l+1) / 2^{n}} L_{k}\left(2^{n+1} x-2 l-1\right) \mathrm{d} x\right|\right] \\
& \leq 2^{-(n+1) \alpha} \sqrt{2 k+1}+\left|f\left((2 l+1) / 2^{n+1}\right)\right|\left|\int_{l / 2^{n}}^{(l+1) / 2^{n}} \phi_{k, n l}(x) \mathrm{d} x\right| \\
& = \begin{cases}2^{-(n+1) \alpha} \sqrt{2 k+1}, & k \neq 0, \\
2^{-(n+1) \alpha}+2^{-n} \sqrt{2}\left|f(2 l+1) / 2^{n}\right|, \quad k=0, & \leq T_{k f l} 2^{-v}, \quad v=\min \{(n+1) \alpha, n\}\end{cases}
\end{aligned}
$$

which completes this proof.

Remark: The upper bounded of Legendre wavelet transform coefficients vanish with exponent in terms of multiplies of the scale index or exponential $\alpha$-Hölder continuity.

Theorem 2. Let $f \in C^{N+\alpha}([0,1])$, suppose that wavelet has $n$ vanishing moments, then the upper bounded estimate of wavelets $\psi(x)$ transform coefficients $k$ such that then the upper bounded estimate of Legendrewavelet transform coefficients satisfies

$$
\left|s_{k, n l}\right| \leq T(k, f) 2^{-v}, \quad v=\min \{(n+1)(N+\alpha), n(N+1)\},
$$

where $T_{k f l}$ is a constant with respect to $k, f$ and $l$.

Proof. The proof of this theorem utilizes the $k-1$ vanishing moments of Legendre wavelet $\phi_{k, n l}$ and Taylor expansion of the function $f$ and then is similar to that of the theorem 1.

Now, taking advantage of the results of (9), (13) and theorem 1, we can derive the upper bounded estimation by the norm $\|\cdot\|_{2}$.

Theorem 3. Let $f \in C^{\alpha}([0,1])$, then the upper bounded estimate of approximation error by using Legendre wavelet bases satisfies

$$
\left\|f-S^{\mathrm{T}} \Phi(x)\right\|_{2}<T \cdot 2^{-2 \min \left\{\left(N_{0}+1\right) \alpha, N_{0}\right\}}+\varepsilon,
$$

where $T$ is a constant with respect to $T_{k f l}$ and $\varepsilon$ is an arbitrarily small positive constant.

Proof. From the equality $\|f\|_{2}=\lim _{n \rightarrow \infty} \sum_{k=0}^{\infty} \sum_{l=0}^{\infty}\left(s_{k, n l}\right)^{2}$, there exists positive integers $N_{0}, N_{1}, K, K_{1}$ and arbitrarily small positive constant $\varepsilon$ satisfying

$$
\begin{aligned}
\left\|f-S^{\mathrm{T}} \Phi(x)\right\|_{2} & =\sum_{n=N}^{\infty} \sum_{k=K}^{\infty} \sum_{l=0}^{\infty}\left(s_{k, n l}\right)^{2}<\sum_{n=N}^{N_{1}} \sum_{k=K}^{K_{1}} \sum_{l=0}^{2^{N_{1}}}\left(s_{k, n l}\right)^{2}+\varepsilon \\
& \leq \sum_{n=N_{0}}^{N_{1}} 2^{-2 v} \cdot \sum_{k=K}^{K_{1}} \sum_{l=0}^{2^{N_{1}}}\left(T_{k f l}\right)^{2}+\varepsilon<T \cdot 2^{-2 \min \left\{\left(N_{0}+1\right) \alpha, N_{0}\right\}}+\varepsilon
\end{aligned}
$$

which completes the proof.

Similarly, we can obtain the estimate of approximation error in space $C^{N+\alpha}([0,1])$.

Theorem 4. Let $f \in C^{N+\alpha}([0,1])$, the upper bounded estimate of approximation error using Legendre wavelet is described as

$$
\left\|f-S^{\mathrm{T}} \Phi(x)\right\|_{2}<T \cdot 2^{-2 \min \left\{\left(N_{0}+1\right)(N+\alpha), N_{0}(N+1)\right\}}+\varepsilon,
$$

where $T$ is a constant with respect to $T_{k f l}$ and $\varepsilon$ is an arbitrarily small positive constant.

These estimates of the approximation error upper bounded provide computational precision for numerical computation. 


\subsection{Approximation Error Estimate by Norm \|\|$_{1}$}

In this subsection, we derive the estimations of approximation error by norm \|\|$_{1}$.

Theorem 5. Let $f \in C^{\alpha}([0,1])$, then the estimation of the approximation error upper bounded by the norm $\|\cdot\|_{1}$ satisfies

$$
\left\|f(x)-S^{\mathrm{T}} \Phi(x)\right\|_{1} \leq T \cdot 2^{-\left(v+N_{0}\right)}+\varepsilon, v=\min \left\{\left(N_{0}+1\right) \alpha, N_{0}\right\},
$$

where $T$ is a constant with respect to $T_{k f l}$ and $\varepsilon$ is an arbitrarily small positive constant.

Proof. Taking advantage of the definition of norm $\|\cdot\|_{1}$ and using (13), it is clear that the approximation error upper bounded is estimated by

$$
\begin{aligned}
\left\|f(x)-S^{\mathrm{T}} \Phi(x)\right\|_{1} & =\int_{0}^{1}\left|f(x)-\sum_{k=0}^{p-1} \sum_{l=0}^{2^{N_{0}}-1} \phi_{k, N_{0} l}(x)\right| \mathrm{d} x=\int_{0}^{1}\left|\sum_{k=0}^{p-1} \sum_{n=N_{0}}^{\infty} \sum_{l=0}^{\infty} s_{k, n l} \phi_{k, n l}(x)\right| \mathrm{d} x \\
& \leq \sum_{n=N_{0}}^{N_{1}} \sum_{k=0}^{p-1} \sum_{l=0}^{2^{N_{1}}-1} \int_{l / 2^{n}}^{(l+1) / 2^{n}}\left|S_{k, n l} \phi_{k, n l}(x)\right| \mathrm{d} x+\varepsilon \\
& \leq \sum_{n=N_{0}}^{N_{1}} \sum_{k=0}^{p-1} \sum_{l=0}^{2^{N_{1}}-1}\left(\left|s_{k, n l}\right| \sqrt{4 k+2}\right)+\varepsilon \leq T \cdot 2^{-\left(v+N_{0}\right)}+\varepsilon .
\end{aligned}
$$

For $f \in C^{N+\alpha}([0,1])$, the estimate technique by the norm \|\|$_{1}$ is similar to the theorem 4 and theorem 5 .

\section{Conclusion}

As all, this paper considers the compactly supported, polynomial, orthogonal and bounded properties of Legendre wavelet bases. Using these properties, the upper bounded estimates of the approximation error are presented for the function belonging to exponential $\alpha$-Hölder continuity and space $C^{N+\alpha}([0,1])$ by norms $\|\cdot\|_{2}$ or $\|\cdot\|_{1}$, respectively.

\section{Acknowledgements}

This work is funded by Fundamental and Advanced Research Project of Chongqing CSTC of China, the project No. are CSTC2013JCYJA00022 and CSTC2012jjA00018.

\section{References}

[1] Beylkin, G. (1992) On the Representation of Operators in Bases of Compactly Supported Wavelets. SIAM Journal on Numerical Analysis, 6, 1716-1739.

[2] Alpert, B. (1993) A Class of Bases in $L_{2}$ for the Sparse Representation of Integral Operators. SIAM Journal on Mathematical Analysis, 24, 247-262.

[3] Maleknejad, K., Aghazadeh, N. and Molapourasl, F. (2006) Numerical Solution of Fredholm Integral Equation of the First Kind with Collocation Method and Estimation of Error Bound. Applied Mathematics and Computation, 179, 352359. http://dx.doi.org/10.1016/j.amc.2005.11.159

[4] Pinheiro, A. and Vidakovic, B. (1997) Estimating the Square Root of a Density via Compactly Supported Wavelets. Computational Statistic and Data Analysis, 25, 399-415. http://dx.doi.org/10.1016/S0167-9473(97)00013-3

[5] Dahmen, W., Kurdila, J.A. and Oswald, P. (1997) Multiscale Wavelet Methods for Partial Differential Equations. Academic Press, San Diego.

[6] Goghary, H. and Goghary, M. (2006) Two Computational Methods for Solving Linear Fredholm Fuzzy Integral Equation of the Second Kind. Applied Mathematics and Computation, 182, 791-796. http://dx.doi.org/10.1016/j.amc.2006.04.039

[7] Maleknejad, K. and Sohrabi, S. (2007) Numerical Solution of Fredholm Integral Equation of the First Kind by Using Legendre Wavelets. Applied Mathematics and Computation, 186, 837-843.

[8] Yousefi, S. and Razzaghi, M. (2005) Legendre Wavelets Method for the Nonlinear Volterra-Fredholm Integral Equations. Mathematics and Computers in Simulation, 70, 1-5. http://dx.doi.org/10.1016/j.matcom.2005.02.035

[9] Razzaghi, M. and Yousefl, S. (2000) Legendre Wavelets Direct Method for Variational Problems. Mathematics and Computers in Simulation, 53, 190-190. 
[10] Razzaghi, M. and Yousefi, S. (2001) Legendre Wavelets Method for the Solution of Nonlinear Problems in the Calculus of Variations. Mathematical and Computer Modelling, 34, 45-54. http://dx.doi.org/10.1016/S0895-7177(01)00048-6

[11] Zheng, X., Yang, X., Su, H. and Qiu, L. (2011) Discontinuous Legendre Wavelet Element Method for Elliptic PartialDifferential Equations. Applied Mathematics and Computation, 218, 3002-3018. http://dx.doi.org/10.1016/j.amc.2011.08.045

\section{Responses to Reviewers' Comments}

Firstly, the authors are grateful to the editors and referees for their valuable comments that greatlyimprove the quality of this paper. We now present responses to the valuable comments proposed by the referees detail by detail.

Common comments proposed by the reviewers.

\section{Comment to the author}

The author introduces Legendre wavelet bases and derives their rich properties.

Here are some comments to this work:

1. In the proof of Lemma 3, it is noted that it should be $\int_{l / 2^{n}}^{(l+1) / 2^{n}} \phi_{1, n l}(x) \mathrm{d} x$, but not $\int_{l / 2^{n}}^{(l+1) / 2^{n}} \phi_{0, n l}(x) \mathrm{d} x$ when $k$ $=0$.

2. In the proof of Lemma 4 , the author should clearly indicate why $(2 k+1)^{1 / 2}\left(2 k^{\prime}+1\right)^{1 / 2}=0$ holds.

3. In page 4, is there something wrong for defining the function $S_{k, n l}$ ? It is noted that the author claims that $f(x)=\lim _{n \rightarrow \infty} \sum_{k=0}^{\infty} \sum_{l=0}^{\infty} s_{k, n l} \phi_{k, n l}(x)$. However, he also defines $S_{k, n l}=\left(f(x), \phi_{k, n l}(x)\right)$. Does it look like a cycle?

4. In the proof of Theorem 1, why does the author emit the part $L_{k}\left(2^{n+1} x-2 l-1\right)$ related to $\int_{l / 2^{n}}^{(l+1) / 2^{n}}\left|f(x)-f\left((2 l+1) / 2^{n+1}\right)\right| \mathrm{d} x$ ? Will the inequality still hold if doing so?

\section{Response}

1. We discuss the situation $k=0$, thus it is $\int_{l / 2^{n}}^{(l+1) / 2^{n}} \phi_{0, n l}(x) \mathrm{d} x$.

2. Because $\int_{-1}^{1} L_{k}(v) L_{k^{\prime}}(v) \mathrm{d} v=0$.

3. $s_{k, n l}$ is the wavelet coefficient and it is calculated by inner product.

4. Because $\phi_{k, n l}(x)=2^{n / 2} \sqrt{2 k+1} L_{k}\left(2^{n+1} x-2 l-1\right)$.

Based on the above responses, I think there is no need to be corrected. 